\title{
Investigations of polypeptide biosynthesis: formation of peptide amides
}

\author{
Shawn E. Ramer, Hengmiao Cheng, and John C. Vederas* \\ Department of Chemistry, University of Alberta, Edmonton, Alberta, Canada T6G 2G2.
}

\begin{abstract}
Peptides having a primary amide functionality at the carboxyl terminus are formed by oxidative cleavage of two carbons from glycine-extended precursors by peptidylgiycine $\alpha$-amidating monooxygenase (PAM) in the presence of oxygen, copper and ascorbic acid. This enzyme was purified from porcine pituitary, and the stereochemistry and mechanism of 1 ts oxidation of D-tyrosyl-L-valylglycine 1 to Dtyrosyl-I-valinamide 2 and glyoxylate 3 was investigated. A new assay was developed based on reaction of glyoxylate with nitrosobenzene to give $\mathrm{N}$-hydroxyformanilide 4 . $\mathrm{R}-$ and $\mathrm{S}-\left[2-{ }^{3} \mathrm{H}\right]$ glycines were prepared by reduction of optically pure 4-benzy loxycarbony 1-3-bromo-5, 6-dipheny 1-2, 3, 5, 6-tetrahydro-1, 4-oxazin-2-ones with tritium gas in [ ${ }^{3}$ ] water. Their stereochemical purity was determined by ${ }_{\mathrm{H}-}$ decoupled tritium NMR of their (1S)-(-)-camphanamide derivatives and by a modified D-amino acid oxidase assay which employs nitrosobenzene to capture glyoxylate. Conversion of the stereospecifically labeled $\left[2-{ }^{3} \mathrm{H}\right]$ glycines to corresponding $\mathrm{D}-$ tyrosyl-L-valyl-[2-3 H]glycines and subsequent PAM oxidation demonstrated that the pro-s hydrogen of the glycine residue is removed. The possible mechanism of PAM and the significance of these results for determining substrate specificity and for designing inhibitors or drugs are discussed.
\end{abstract}

\section{INTRODUCTION}

Peptides having a primary amide functionality at the carboxyl terminus are widely distributed in animals and elicit important physiological effects (ref, 1,2). Examples include compounds such as vasopressin from the pituitary, lutenizing hormone releasing hormone (LHRH) from the hypothalamus, gastrins from the gastric antrum, and substance $P$ from the spinal ganglia. In the cases studied so far, the peptide amides are generated by posttranslational stepwise cleavage of larger precursor proteins (ref. 3,4). These proteins generally have a glycyl residue attached to the amino acid which will become the carboxy terminal amide. In 1982 an enzyme was detected in porcine pituitary which is able to cleave a synthetic tripeptide, D-tyrosyl-L-valylglycine 1 , to D-tyrosyl-L-valinamide $\underline{2}$ and glyoxylate $\underline{3}$ (Fig. 1, ref. 5). Subsequently it was shown that this enzyme, peptidyl<smiles>CC(C)[C@H](NC(=O)[C@H](N)CC1CC=C(O)CC1)C(=O)N[C@@H](C)C(=O)O</smiles>

1<smiles>CC(C)[C@H](NC(=O)[C@@H](N)CC1CC2CCC(O)(CC2)C1)C(N)=O</smiles>

a $\quad={ }^{14} \mathrm{C}$

b $H_{S}=I_{H}$ $H_{\mathbf{R}}=\mathbf{3}_{\mathbf{H}}$

C $H_{S}=3_{H}$ $H_{R}=1_{H}$

d $H_{S}={ }^{3} H, 1_{H} \quad H_{R}=1_{H}, 3_{H}$

Fig. 1 glycine $\alpha$-amidating monooxygenase (PAM), requires copper, oxygen and ascorbate, occurs in multiple forms (ref, 6), and is present in a host of mammalian tissues (ref. 7) as well as in frogs (ref. 8). since all published assays for the PAM enzyme (s) rely on detection of a specific product amide (e.g., radioimmunoassay), they are limited to unique substrates and do not permit examination of events at the carbons and hydrogens of the glycine residue. The present study describes: a new assay for PAM based on capture of glyoxylate; preparation of $\mathrm{R}-$ and $\mathrm{S}-$ $\left[2-{ }^{3}\right]$ glycines and new methods for determination of their stereochemical purity; and the stereochemistry of hydrogen removal from 1 by PAM. The resulting information aids $\overline{i n}$ understanding the steric requirements and mechanism of PAM and may be useful in design of inhibitors or orallyactive peptide hormone prodrugs. 


\section{GLYOXYLATE-BASED PAM ASSAY}

Most published procedures for detection of glyoxylate in biochemical systems appeared to lack the necessary sensitivity or specificity and frequently required extensive sample preparation. Hence an alternative assay was developed based on the unusual and highly specific reaction of glyoxylic acid 3 with nitrosobenzene under aqueous conditions to form $\mathrm{N}$-hydroxyformanilide $\underline{4}$ (Fig. 2 , ref. 9 ). The product 4 is formed reproducibly $( \pm 2 \%)$ in

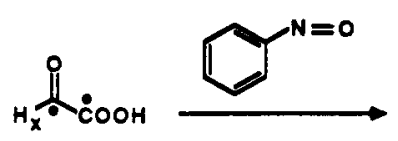

3

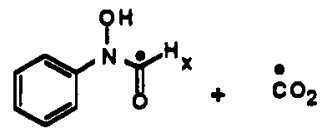

4 high yield and is easily extracted from the aqueous media with ethyl ether. Control experiments in deuteriated water showed that the reaction does not exchange the formyl hydrogen of $\underline{3}$ or $\underline{4}$ with solvent.

Fig. 2

Radioisotopic labeling was used to obtain the necessary sensitivity for potentially small amounts of PAM enzyme and glyoxylate production. The tripeptide $1 \mathrm{a}$, in which both glycine carbons are carbon-14, was synthesized by standard solution phase methods from $\left[1,2-{ }^{14} \mathrm{C}_{2}\right]$ glycine $5 \mathrm{a}(113 \mathrm{mCi} / \mathrm{mmol}, 96 \%$

$\left.{ }^{14} \mathrm{C}\right)$. The PAM enzyme was isolated by a modified literature procedure (ref, 10) which employs affinity chromatography with unlabeled tripeptide 1 bound to Affi-Gel 15 to give protein of an estimated 90-95\% purity. The enzyme was assayed by incubation with the radioactive tripeptide $1 \mathrm{a}$ in the presence of oxygen, ascorbate, copper sulfate, and potassium iodide at $37^{\circ} \mathrm{C}$. Radioactive glyoxylate $3 a$ formed by this reaction was trapped by diflution with unlabeled glyoxylic acid and addition of excess nitrosobenzene to generate $14 \mathrm{C}-1$ abeled $\mathrm{N}$-hydroxyformanilide $4 \mathrm{a}$. The latter was extracted with ether, dried, and analyzed by scintillation counting. A variety of isotope dilution and control experiments confirmed that the radioactivity resides in $4 a$ and is due to PAM-catalyzed conversion of the labeled tripeptide $1 \mathrm{a}$. This new assay will allow rapid comparison of the activities of various PAM enzymes toward a large variety of substrates bearing a carboxy terminal glycine. Hence this may aid assignment of a particular biological function to different forms of the enzyme. In addition, this assay permits examination of the stereochemistry of hydrogen loss from the glycyl residue during PAM oxidation of tripeptide 1 .

\section{SYNTHESIS AND ANALYSIS OF STEREOSPECIFICALLY LABELLED $\left[2{ }^{3} \mathrm{H}\right]$ GLYCINES}

To determine the stereospecificity of hydrogen removal by PAM, it was essential to obtain chiral samples of glycine bearing tritium labels in the pro $R$ and pro $S$ positions. Most previously-reported chemical syntheses of stereospecifically deuteriated or tritiated glycines employ multiple steps and introduce label early in the sequence. Although enzymatic exchange of the glycine hydrogen with solvent using serine hydroxymethyltransferase or alanine aminotransferase requires only a single step, such approaches can be problematic due to excessive non-enzymatic or incomplete exchange. Since the elegant method of williams and coworkers for synthesis of stereospecifically deuteriated glycines

introduces label in the final stage (ref. 11), it was adapted for use with tritium gas at one atmosphere pressure. Bromination of the $(5 R, 6 S)$ oxazinone 6 and its enantiomer 7 with $\mathrm{N}$-bromosuccinimide gave the bromo compounds $\underline{8}$ and $\underline{9}$, respective $\bar{l} y$ (Fig. 3 ). Hydrogenation of these with pure tritium gas and palladium chloride catalyst in a mixture of tritiated water $(50 \mathrm{Ci} / \mathrm{mL}, 0.91 \mathrm{Ci} / \mathrm{mmol})$ and tetrahydrofuran produced (R) $-\left[2-{ }^{3} \mathrm{H}\right]$ glycine $5 \mathrm{~b}$ and its $\mathrm{s}-$ isomer $5 c$, respectively. The yields of $5 b$ and $5 c$ after HPLC purification were $28 \%$ and $31 \%$ and the specific activities were 1.0 and $0.78 \mathrm{ci} / \mathrm{mmol}$, respectively. The stereochemical

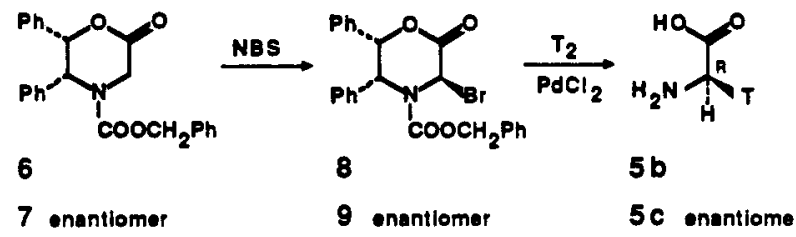

Fig. 3

purity of these glycines was analyzed by two methods. In the first approach, the tritiated glycines $5 b$ and $5 c$ were converted to their corresponding (1s)-(-)-camphanamide derivatives (ref. 12) which were examined by $320 \mathrm{MHz} H$-decoupled H-NMR spectrometry. Integration of the signals showed that 938 of the tritium in the camphanamide of $5 \mathrm{~b}$ was in the $\mathrm{R}$ position (upfield) whereas in the corresponding derivative of $5 \mathrm{c}$ it was $88 \%$ in the $s$ position (downfield) (Fig. 4). The complete absence $(51 \%)$ of doubly-labeled ( $\left[{ }^{3} \mathrm{H}_{2}\right.$ ) glycine camphanamide) species was evident from lack of signals due to tritium-tritium coupling. Clearly tritium NMR spectrometry can be an effective alternative to enzymatic analysis for 


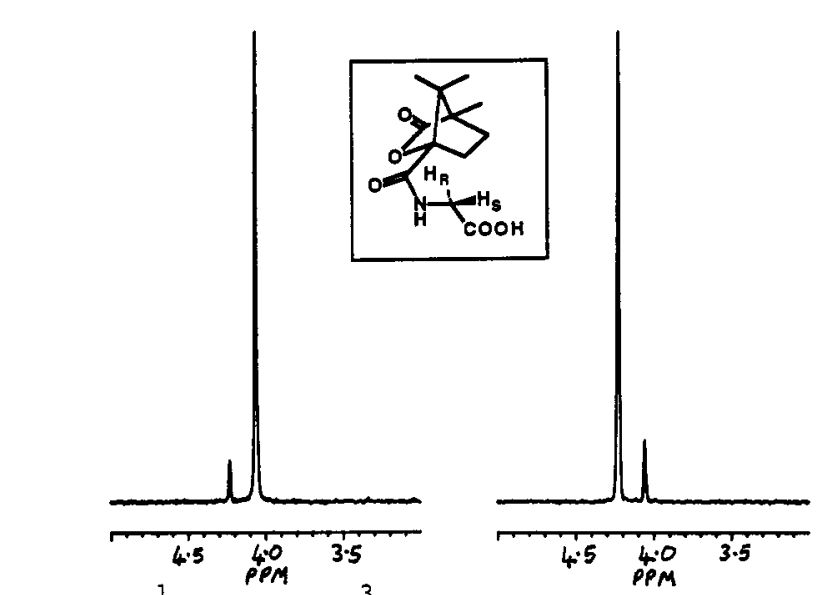

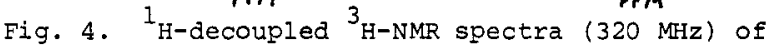
camphanamides of $\underline{5 b}$ (left) and $5 \mathrm{c}$ (right). determination of tritium stereochemistry, provided that a sufficient level of label is present.

A common enzymatic determination of glycine stereochemistry employs Damino acid oxidase to release the pro $S$ hydrogen into the aqueous media with concomitant formation of glyoxylic acid (ref. 13,14). The water is then isolated and its isotopic content is determined. Its major limitations are the requirements for quantitative water capture and for complete oxidation of all glycine because of the primary isotope effect for 5 labeled molecules. These difficulties can be avoided by addition of $[1,2-$ ${ }^{4} \mathrm{C}_{2}$ ]glycine $5 \mathrm{a}$ to samples of $5 \mathrm{~b}$ or $5 \mathrm{c}$ prior to exposure to D-amino acid oxidase, and then capture of resulting labeled glyoxylate using the nitrosobenzene procedure. During this process half of the carbon-14 is lost as $\mathrm{CO}_{2}$, all of the initial tritium in the $\mathrm{R}-\left[2-{ }^{3} \mathrm{H}\right]-$ glycine $5 b$ is retained, and all of the initial tritium of any $s-[2-3 \mathrm{H}]$ glycine $5 c$ which is oxidized is lost (Fig. 5). Hence the $3 \mathrm{H} /{ }^{1} \mathrm{C}$ ratio of the $\mathrm{N}$-hydroxyformanilide will be twice the initial glycine ratio for the R-isomer, it will go to zero for the S-isomer, and it will

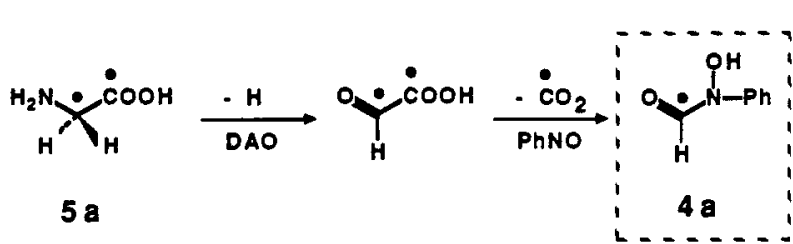

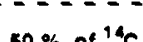
stay constant for a racemic mixture. This new assay is transparent for the primary tritium isotope effect (provided that doubly-tritiated species are absent) because the product of the $S$ isomer is not radioactive. Our results show that the combined effects of the secondary tritium isotope effect during the enzymatic step and the primary carbon14 isotope effect during the nitrosobenzene reaction are relatively small (< 5\%). Hence this assay gives accurate results with only partial conversion and eliminates the need for quantitative water entrapment. Analysis of $5 b$ and $5 c$ gave values in good agreement with those obtained by ${ }^{3}$-NMR spectra of their camphanamides.

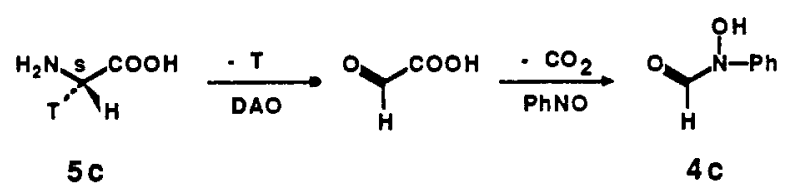

DAO a D-Amino Acid Oxidase

$0 \%$ of ${ }^{3} \mathrm{H}$

Fig. 5. Fate of labeled atoms $\left(\bullet={ }^{14} \mathrm{C}, \mathrm{T}=3_{\mathrm{H}}\right)$ during

D-amino acid oxidase/nitrosobenzene assay.

\section{STEREOCHEMISTRY AND MECHANISM OF PAM OXIDATION}

The R-, S-, and RS- $\left[2-{ }^{3} \mathrm{H}_{1}\right]$ glycines $\underline{5 b}, \underline{5 c}$, and $\underline{5 d}$ were individually mixed with $\left[1,2-{ }^{14} C_{2}\right]-$ glycine $5 \mathrm{a}$ and transformed to the corresponding $\mathrm{D}$-tyrosyl-L-valylglycines $1 \mathrm{~b}$, $1 \mathrm{c}$, and $1 \mathrm{~d}$, respectively, by standard solution phase peptide synthesis methods. These tripeptides were then exposed to the purified PAM from porcine pituitary, the radioactive glyoxylates were trapped with nitrosobenzene, and the $3 \mathrm{H} /{ }^{14} \mathrm{C}$ ratios of the resulting $\mathrm{N}$-hydroxyformanilides were compared to those of the starting tripeptides. The results clearly show loss of the pro $S$ hydrogen of the glycine restidue of 1 and retention of the pro $R$ hydrogen during the PAM reaction. The changes in ${ }_{\mathrm{H}} /{ }^{4} \mathrm{C}$ ratio were not as complete as initially expected because of some partial epimerization during chemical synthesis of the tripeptides 1 . This was confirmed by hydrolysis of the tripeptides $1 \mathrm{~b}$ and $1 \mathrm{c}$ to regenerate glycines and stereochemical analysis of these by the D-amino acid oxidase/ nitrosobenzene procedure. This showed that as expected, the PAM oxidation is completely stereospecific. 
Initially it was proposed that PAM dehydrogenates the glycine residue of $A$ to an $N$-acyl imine $C_{j}$ this adds water to form $B$ which then cleaves to the peptide amide and glyoxylate (Fig. $\overline{6}$, ref. 5). A new more generally-accepted mechanism involves direct hydroxylation of

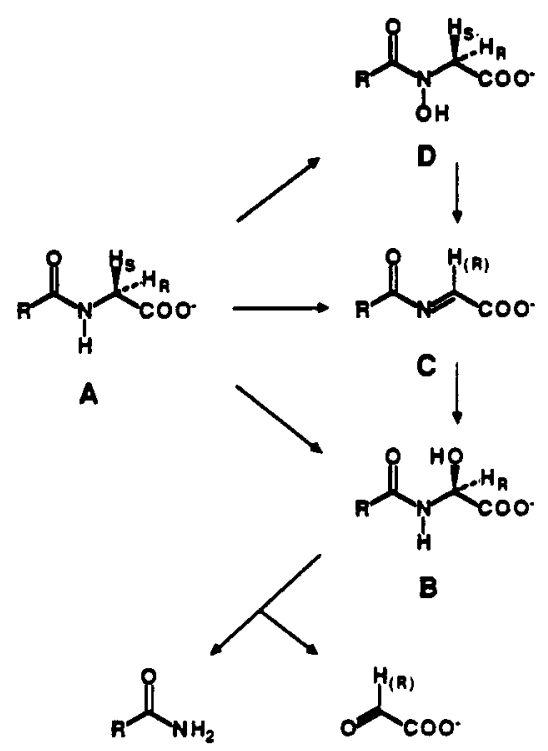

Fig. 6. Possible mechanisms of PAM oxidation.

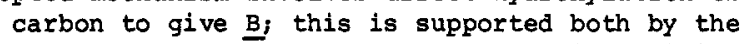
cofactor requirements of PAM and by its ability to transform glyoxylic acid phenylhydrazone to oxalic acid monohydrazide (ref, 15). Although less likely, another possibility may be $\mathrm{N}-$ hydroxylation to generate $D$ followed by transformation to $\mathrm{C}$ and $\mathrm{B}$. Lead tetraacetate oxidation of $\mathrm{N}$-aroylglycines provides a chemical precedent for this sequence (ref. 16). These possibilities may be distinguished using an analog of 1 in which the glycine nitrogen is replaced with oxygen (i.e., a glycolate ester); only a direct c-hydroxylation mechanism should be capable of forming glyoxylate. Such compounds have been synthesized and their interaction with PAM is under investigation. since enzymatic c-hydroxylations generally proceed with retention of configuration (ref. 17), the removal of the pro $s$ hydrogen by this process would require that aminal $B$ possess $S$ configuration. This suggests that peptide analogs that bear a hydroxyl in that position but are incapable of cleavage may be inhibitors of PAM; this is in accord with experimental observations (ref. 10). The stereochemistry of PAM oxidation clearly shows why this enzyme will not permit replacement of the glycine residue with I-amino acids but will accept a terminal

D-alanine residue (ref. 18). Since a terminal D-amino acid is likely to afford some protection against peptidase cleavage, peptide hormones bearing such extensions possess potential to be orally-active prodrugs. Investigation of this possibility and further studies on PAM enzymes are continuing.

Acknowledgements We thank Hiromi Morimoto, Philip G. Williams, and Albert Dorsky (Lawrence Berkeley Laboratory) for expert technical assistance. We are indebted to Robert M. Williams (Colorado State University) for generous gifts of oxazines and helpful discussions. These investigations were supported by the Natural Sciences and Engineering Research Council of Canada, the Alberta Heritage Foundation for Medical Research, and the University of Alberta Central Research Fund.

\section{REFERENCES}

1. W. Voelter and G. Weitzel, Structure and Activity of Natural Peptides, Walter de Gruyter, New York (1981).

2. G. Kreil, Methods Enzymol. 106, 218-223 (1984).

3. R.E. Mains, B.A. Eipper, C.C. Glembotski, and R.M. Dores, Trends Neurosci. Pers. Ed. 6, 229-235 (1983).

4. J. Van Nispen and R. Pinder, Annu. Rep. Med. Chem. 21, 51-62 (1986).

5. A.F. Bradbury, M.D.A. Finnie, and D.G. Smyth, Nature 298, 686-688 (1982).

6. A.S.N. Murthy, R.E. Mains, and B.A. Eipper, J. Biol. Chem. 261, 1815-1822 (1986).

7. J. Sakata, K. Mizuno, and H. Matsuo, Biochem. Biophys. Res. Commun. 140, 230-236 (1986).

8. C. Mollay, J. Wichta, and G. Kreil, FEBS Lett. 202, 251-254 (1986).

9. M.D. Corbett and B.R. Corbett, J. Org. Chem. 45, 2834-2839 (1980).

10. J.S. Kizer, R.C. Bateman, J.H. Miller, W.H. Busby, and W.W. Youngblood, Endocrinology $118,2262-2267$ (1986).

11. R.M. Williams, D. Zhai, and P.J. Sinclair, J. Org. Chem. 51, 5021-5022 (1986).

12. W.L.F. Armarego, B.A. Milloy, and W. Pendergast, J. Chem. Soc., Perkin Trans. 1, 22292237 (1976).

13. D. Wellner, Biochemistry 9, 2307-2310 (1970).

14. A.G. Palekar, S.S. Tate, and A. Meister, Biochemistry 9, 2310-2315 (1970).

15. A.F. Bradbury and D.G. Smyth, Eur. J. Biochem. 169, 579-584 (1987).

16. A.P. Gledhill, C.J. McCall, and M.D. Threadgili, J. Org. Chem. 51, 3196-3201 (1986).

17. S. Shapiro, J.U. Piper, and E. Caspi, J. Am. Chem. Soc. 104, 2301-2305 (1982).

18. A.E.N. Landymore-Lim, A.F. Bradbuxy, and D.G. Smyth, Biochem. Biophys. Res. Commun. $117,289-293(1983)$. 